\title{
IMPLICIT BLOCK HYBRID-LIKE METHOD FOR SOLVING SYSTEM OF FIRST ORDER ORDINARY DIFFERENTIAL EQUATIONS
}

\author{
Lee Ken Yap ${ }^{1,2^{*}}$, Fudziah Ismail ${ }^{1}$ \\ ${ }^{1}$ Universiti Putra Malaysia, 43400 UPM Serdang, Malaysia. \\ ${ }^{2}$ Universiti Tunku Abdul Rahman, Bandar Sungai Long, 43000 Kajang, Malaysia. \\ *Corresponding Author: 1kyap@utar.edu.my
}

\begin{abstract}
The block hybrid-like method is presented and implemented in predictor-corrector mode to solve system of first order ordinary differential equations. The block method is applied to provide the approximation for both the main and off-step points concurrently. The stability properties of the method are investigated. Some illustrative examples are presented to demonstrate the efficiency of the block hybrid-like method.
\end{abstract}

(Keywords: implicit, block, off-step point, ordinary differential equations)

\section{INTRODUCTION}

We consider the first order ordinary differential equations (ODEs)

$$
y^{\prime}=f(x, y)
$$

with initial condition $y(a)=y_{0}$ for $x \in[a, b]$. Eq. (1) is frequently found in many physical problems. Several researchers have proposed the numerical block methods to solve first order ODEs. The major advantage of the block methods is that in each of its implementation, the solution is approximated at few points concurrently. Ibrahim et al. [9] and Musa et al. [14] suggested the block backward differentiation formula methods for stiff ODEs. Akinfenwa et al. [2] introduced the block hybrid backward differential formula method based on the interpolation and collocation of the basic polynomial. Lee [11] proposed the 2-point and 3-point block methods based on backward difference form for non-stiff ODEs. Omar and Suleiman [15] implemented the parallel 2-point block method of variable step size and order for system of non-stiff ODEs. Mehrkanoon et al. [13] applied the four-point implicit block multistep method for solving system of (1) in variable step size. All these block methods ([9], [13], [14], [15]) estimate the solutions of $y$ at few main points concurrently.

Many researchers have also shown their interest on the block hybrid methods that find the approximation of $y$ at both the main and off-step points simultaneously. In the study of Enright and Higham [7], it demonstrated that the precise location of the off-step points was efficiently used to minimize the interpolation errors and to develop various cheap strategies for step size control. Akinfenwa et al. [3] proposed the implicit two step hybrid block collocation method with four off-steps points. James et al. [10] suggested the one step block hybrid method with five off-step points. Akinfenwa et al. [3] and James et al. [10] applied the interpolation and collocation on basic polynomial to derive the method. However, the implementation is slightly different. Akinfenwa et al. [3] implemented the proposed method as the self starting method that does not require the starting values to proceed while James et al. [10] implemented the proposed method in predictorcorrector mode. Abasi et al. [1] derived the block hybrid method based on backward differentiation formula and interpolation polynomial that interpolates the value of $y$ at two main points and two off-step points.

Here, we are going to apply the block hybrid-like method in predictor-corrector mode to solve system of first order ODEs (1). The implementation of this method approximates the solution of $y$ at the main point $x_{n+1}$ and the off-step point $x_{n+\frac{1}{2}}$ simultaneously.

\section{DERIVATION OF IMPLICIT BLOCK HYBRID- LIKE METHOD}

In this section, we explain the mathematical formulation of the implicit block hybrid-like method that based on numerical integration. The derivation involves the divided differences that relative to both the main and off-step points. 


\section{Main Point Method}

We define the main point as $x_{n+1}=x_{n}+h$ and integrate the first order differential equation (1) from $x_{n}$ to $x_{n+1}$ on both sides, it then leads to

$$
\int_{x_{n}}^{x_{n+1}} y^{\prime}(x) d x=\int_{x_{n}}^{x_{n+1}} f(x, y) d x .
$$

Next, we define the interpolation polynomial to interpolate $f(x, y)$ and substitute in (2)

$$
y\left(x_{n+1}\right)-y\left(x_{n}\right)=\int_{x_{n}}^{x_{n+1}} P_{n}(x) d x
$$

with $s=\frac{x-x_{n+1}}{h}$. By changing the limit of integration and replace $d x=h d s$, we obtain

$$
\begin{aligned}
y\left(x_{n+1}\right)=y\left(x_{n}\right) & +\int_{-1}^{0} P_{n}\left(x_{n+1}+s h\right) h d s \\
=y\left(x_{n}\right) & +h\left(\int_{-1}^{0} 1 d s f\left[x_{n+1}\right]\right. \\
& +h \int_{-1}^{0} s d s f\left[x_{n+\frac{1}{2}}, x_{n+1}\right] \\
& +h^{2} \int_{-1}^{0} s(s \\
& \left.\left.+\frac{1}{2}\right) d s f\left[x_{n}, x_{n+\frac{1}{2}}, x_{n+1}\right]+\cdots\right) .
\end{aligned}
$$

Here, we consider the terms up to $f\left[x_{n}, x_{n+\frac{1}{2}}, x_{n+1}\right]$ with

$f\left[x_{n+1}\right]=f_{n+1} ;$

$f\left[x_{n+\frac{1}{2}}, x_{n+1}\right]=\frac{2}{h}\left(f_{n+1}-f_{n+\frac{1}{2}}\right)$;

$f\left[x_{n}, x_{n+\frac{1}{2}}, x_{n+1}\right]=\frac{2}{h^{2}}\left(f_{n+1}-2 f_{n+\frac{1}{2}}+f_{n}\right)$.

After simplified, we obtain

$$
y_{n+1}=y_{n}+h\left(\frac{1}{6} f_{n+1}+\frac{2}{3} f_{n+\frac{1}{2}}+\frac{1}{6} f_{n}\right) .
$$

\section{Off-step Point Method}

Similarly, we define the off-step point as $x_{n+\frac{1}{2}}=x_{n}+$ $\frac{1}{2} h$. Integrate (1) from $x_{n}$ to $x_{n+\frac{1}{2}}$ and replace $f(x, y)$ with interpolation polynomial gives

$$
\begin{aligned}
y\left(x_{n+\frac{1}{2}}\right)=y\left(x_{n}\right) & \\
& +h\left(\int_{-\frac{1}{2}}^{0} 1 d s f\left[x_{n+\frac{1}{2}}\right]\right. \\
& +h \int_{-\frac{1}{2}}^{0} s d s f\left[x_{n}, x_{n+\frac{1}{2}}\right] \\
& +h^{2} \int_{-\frac{1}{2}}^{0} s(s \\
& \left.\left.+\frac{1}{2}\right) d s f\left[x_{n-\frac{1}{2}}, x_{n}, x_{n+\frac{1}{2}}\right]\right)
\end{aligned}
$$

where $s=\frac{x-x}{h+\frac{1}{2}}$ and

$f\left[x_{n+\frac{1}{2}}\right]=f_{n+\frac{1}{2}} ;$

$f\left[x_{n}, x_{n+\frac{1}{2}}\right]=\frac{2}{h}\left(f_{n+\frac{1}{2}}-f_{n}\right)$;

$f\left[x_{n-\frac{1}{2}}, x_{n}, x_{n+\frac{1}{2}}\right]=\frac{2}{h^{2}}\left(f_{n+\frac{1}{2}}-2 f_{n}+f_{n-\frac{1}{2}}\right)$.

After simplified, we obtain

$$
y_{n+\frac{1}{2}}=y_{n}+h\left(\frac{5}{24} f_{n+\frac{1}{2}}+\frac{1}{3} f_{n}-\frac{1}{24} f_{n-\frac{1}{2}}\right)
$$

We combine the main point method (3) and the off-step point method (4) as the implicit block hybrid-like method.

\section{THE PREDICTOR}

Let the off-step and main points be defined as $x_{n+\frac{1}{2}}=$ $x_{n}+\frac{1}{2} h$ and $x_{n+1}=x_{n}+h$, respectively. Similar technique has been applied to obtain the explicit method as the predictor. It leads to

$$
\begin{aligned}
y\left(x_{n+\frac{1}{2}}\right)=y\left(x_{n}\right) & \\
& +h\left(\int_{0}^{\frac{1}{2}} 1 d s f\left[x_{n}\right]\right. \\
& +h \int_{0}^{\frac{1}{2}} s d s f\left[x_{n-\frac{1}{2}}, x_{n}\right] \\
& +h^{2} \int_{0}^{\frac{1}{2}} s(s \\
& \left.\left.+\frac{1}{2}\right) d s f\left[x_{n-1}, x_{n-\frac{1}{2}}, x_{n}\right]\right)
\end{aligned}
$$


and

$$
\begin{aligned}
y\left(x_{n+1}\right)=y\left(x_{n}\right) & +h\left(\int_{0}^{1} 1 d s f\left[x_{n}\right]\right. \\
& +h \int_{0}^{1} s d s f\left[x_{n-\frac{1}{2}}, x_{n}\right] \\
& +h^{2} \int_{0}^{1} s(s \\
& \left.\left.+\frac{1}{2}\right) d s f\left[x_{n-1}, x_{n-\frac{1}{2}}, x_{n}\right]\right)
\end{aligned}
$$

with $s=\frac{x-x_{n}}{h}$. Substitute the divided difference with

$f\left[x_{n}\right]=f_{n} ;$

$f\left[x_{n-\frac{1}{2}}, x_{n}\right]=\frac{2}{h}\left(f_{n}-f_{n-\frac{1}{2}}\right)$;

$f\left[x_{n-1}, x_{n-\frac{1}{2}}, x_{n}\right]=\frac{2}{h^{2}}\left(f_{n}-2 f_{n-\frac{1}{2}}+f_{n-1}\right)$.

After simplified, we develop the explicit block hybridlike method as follows

$$
\begin{aligned}
& y_{n+\frac{1}{2}}=y_{n}+h\left(\frac{23}{24} f_{n}-\frac{2}{3} f_{n-\frac{1}{2}}+\frac{5}{24} f_{n-1}\right) \\
& y_{n+1}=y_{n}+h\left(\frac{19}{6} f_{n}-\frac{10}{3} f_{n-\frac{1}{2}}+\frac{7}{6} f_{n-1}\right) .
\end{aligned}
$$

\section{STABILITY ANALYSIS}

To analyze the stability property of the implicit block hybrid-like method, we apply the standard test equation $y^{\prime}=\lambda y$ where $\lambda$ is the complex parameter.

$$
\begin{gathered}
y_{n+\frac{1}{2}}=y_{n}+h\left(\frac{5}{24} \lambda y_{n+\frac{1}{2}}+\frac{1}{3} \lambda y_{n}-\frac{1}{24} \lambda y_{n-\frac{1}{2}}\right) \\
y_{n+1}=y_{n}+h\left(\frac{1}{6} \lambda y_{n+1}+\frac{2}{3} \lambda y_{n+\frac{1}{2}}+\frac{1}{6} \lambda y_{n}\right) .
\end{gathered}
$$

It can also be shown in matrix finite difference equation as follows

$$
(I-A h \lambda) Y_{m+1}=(B+C h \lambda) Y_{m}
$$

with

$$
\begin{gathered}
I=\left[\begin{array}{ll}
1 & 0 \\
0 & 1
\end{array}\right], A=\left[\begin{array}{cc}
\frac{5}{24} & 0 \\
\frac{2}{3} & \frac{1}{6}
\end{array}\right], B=\left[\begin{array}{ll}
0 & 1 \\
0 & 1
\end{array}\right], \\
C=\left[\begin{array}{cc}
-\frac{1}{24} & \frac{1}{3} \\
0 & \frac{1}{6}
\end{array}\right], Y_{m+1}=\left[\begin{array}{c}
y_{n+\frac{1}{2}} \\
y_{n+1}
\end{array}\right], Y_{m}=\left[\begin{array}{c}
y_{n-\frac{1}{2}} \\
y_{n}
\end{array}\right] .
\end{gathered}
$$

The stability polynomial is given by

$$
\rho(t)=\operatorname{Det}[(I-A \bar{h}) t-(B+C \bar{h})]
$$

where $\bar{h}=h \lambda$. By taking $\rho(t)=0$, it leads to

$$
\begin{gathered}
\left(1-\frac{3 \bar{h}}{8}+\frac{5 \bar{h}^{2}}{144}\right) \\
t^{2}-\left(1+\frac{7 \bar{h}}{12}+\frac{7 \bar{h}^{2}}{36}\right) t \\
-\left(\frac{\bar{h}}{24}+\frac{\bar{h}^{2}}{144}\right)=0 .
\end{gathered}
$$

The stability polynomial is solved for $\bar{h}$ which gives $|t| \leq 1$ whereby the stability region is obtained by tracing the values of $\bar{h}$. Fig. 1 presents the stability region for the implicit block hybrid-like method. The region of absolute stability lies inside the boundary.

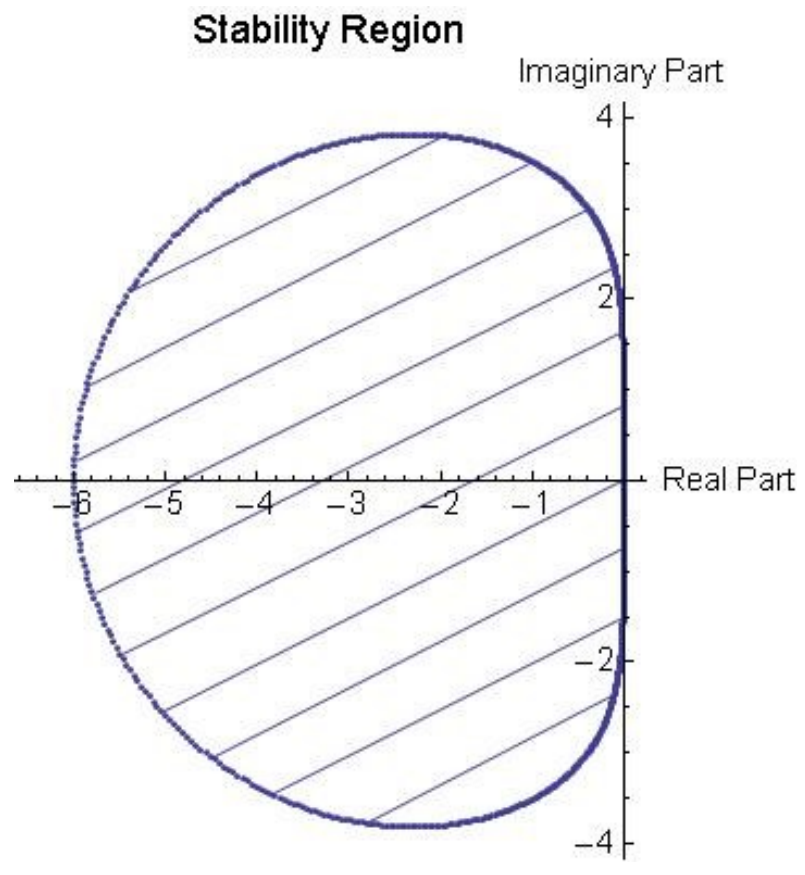

Figure 1. Stability region for implicit block hybrid-like method

By solving $\rho(t)=0$ and $h=0$, the first characteristic polynomial is determined as follows

$$
t^{2}-t=0
$$

Since the roots of the first characteristic polynomial have modulus at most one, this implies the zero stability of the implicit block hybrid-like method. 


\section{NUMERICAL EXAMPLES}

To assert the effectiveness of our approach, we provide the numerical evidence by considering the following test problems.

Problem 1: See [4]

$y_{1}^{\prime}=-y_{1}+y_{2}\left(1-y_{1}-y_{2}\right)$,

$y_{2}^{\prime}=y_{1}-y_{2}\left(1-y_{1}\right)-e^{-x}$,

$y_{1}(1)=\frac{1}{e}, y_{2}(1)=0, x \in[1,2]$

Exact solution: $y_{1}(x)=e^{-x}, y_{2}(x)=0$.

Problem 2: See [5]

$y_{1}^{\prime}=-4 y_{1}+2 y_{2}, y_{1}(1)=\frac{1}{e^{4}}$

$y_{2}^{\prime}=\frac{1}{x^{2}} y_{1}-4 y_{2}, y_{2}(1)=\frac{1}{e^{4}}, x \in[1,5]$;

\section{Exact solution:}

$y_{1}(x)=x^{2} e^{-4 x}, y_{2}(x)=x e^{-4 x}$

Problem 3: See [8]

$y_{1}^{\prime}=\frac{1}{2(1+x)} y_{1}+2 x y_{2}, y_{1}(0)=1$,

$y_{2}^{\prime}=\frac{1}{2(1+x)} y_{2}-2 x y_{1}, y_{2}(0)=0, x \in[0,3]$

Exact solution:

$y_{1}(x)=\sqrt{1+x} \cos x^{2}, y_{2}(x)=\sqrt{1+x} \sin x^{2}$.

Problem 4: See [12]

$$
\begin{aligned}
& y_{1}^{\prime}=y_{3}, y_{1}(0)=1, \\
& y_{2}^{\prime}=y_{4}, y_{2}(0)=1, \\
& y_{3}^{\prime}=-e^{-x} y_{2}, y_{3}(0)=0, \\
& y_{4}^{\prime}=2 e^{x} y_{3}, y_{4}(0)=1, x \in[0, \pi]
\end{aligned}
$$

Exact solution:

$$
\begin{aligned}
& y_{1}(x)=\cos x, y_{2}(x)=e^{x} \cos x \\
& y_{3}(x)=-\sin x, y_{4}(x)=e^{x} \cos x-e^{x} \sin x
\end{aligned}
$$

Problem 5: See [12]

$y_{1}^{\prime}=y_{3}, y_{1}(0)=0, x \in[0,10]$

$y_{2}^{\prime}=y_{4}, y_{2}(0)=1$

$y_{3}^{\prime}=-y_{2}+\sin \pi x, y_{3}(0)=-1$

$y_{4}^{\prime}=-y_{1}+1-\pi^{2} \sin \pi x, y_{4}(0)=1+\pi$

Exact solution:

$y_{1}(x)=1-e^{x}, y_{2}(x)=e^{x}+\sin \pi x$,

$y_{3}(x)=-e^{x}, \quad y_{4}(x)=e^{x}+\pi \cos \pi x$

Problems 1 to 3 are the coupled of first order ODEs while Problems 4 and 5 cover the systems of four first order ODEs. Tables I to V present the numerical results for solving Problems 1 to 5. The explicit and implicit block hybrid-like methods are implemented in predictor-corrector mode to solve these systems in constant step size. The numerical comparison is made between the block hybrid-like method and the existing Adams method. The notations used in the table take the following meaning

$h \quad$ Step size.

Max Maximum error for the computed solution ADM Adams method of order three (See [6]).

I1P Implicit block hybrid-like method of order three. 
Table 1. Numerical Results for Problem

\begin{tabular}{lcc}
\hline$h$ & Method & Max \\
\hline 0.05 & I1P & $2.9220 \times 10^{-8}$ \\
& ADM & $8.2656 \times 10^{-7}$ \\
0.01 & I1P & $4.7153 \times 10^{-11}$ \\
& ADM & $6.5082 \times 10^{-9}$ \\
0.005 & I1P & $2.9512 \times 10^{-12}$ \\
& ADM & $8.1220 \times 10^{-10}$ \\
0.001 & I1P & $4.7275 \times 10^{-15}$ \\
& ADM & $6.4893 \times 10^{-12}$ \\
0.0005 & I1P & $2.9552 \times 10^{-16}$ \\
& ADM & $8.1107 \times 10^{-13}$ \\
0.0001 & I1P & $4.7288 \times 10^{-19}$ \\
& ADM & $8.0214 \times 10^{-15}$ \\
\hline
\end{tabular}

Table 2. Numerical Results for Problem 2

\begin{tabular}{lcc}
\hline$h$ & Method & Max \\
\hline 0.05 & I1P & $4.0107 \times 10^{-7}$ \\
& ADM & $7.5447 \times 10^{-7}$ \\
0.01 & I1P & $2.0327 \times 10^{-10}$ \\
& ADM & $4.3704 \times 10^{-9}$ \\
0.005 & I1P & $1.2746 \times 10^{-11}$ \\
& ADM & $5.2111 \times 10^{-10}$ \\
0.001 & I1P & $2.0456 \times 10^{-14}$ \\
& ADM & $4.0087 \times 10^{-12}$ \\
0.0005 & I1P & $1.2790 \times 10^{-15}$ \\
& ADM & $4.9859 \times 10^{-13}$ \\
0.0001 & I1P & $2.0471 \times 10^{-18}$ \\
& ADM & $4.0107 \times 10^{-15}$ \\
\hline
\end{tabular}

Table 3. Numerical Results for Problem 3

\begin{tabular}{ccc}
\hline$h$ & Method & Max \\
\hline 0.05 & I1P & $2.1118 \times 10^{-3}$ \\
& ADM & $9.6503 \times 10^{-3}$ \\
0.01 & I1P & $3.3559 \times 10^{-6}$ \\
& ADM & $7.1684 \times 10^{-5}$ \\
0.005 & I1P & $2.0889 \times 10^{-7}$ \\
& ADM & $8.6441 \times 10^{-6}$ \\
0.001 & I1P & $3.3294 \times 10^{-10}$ \\
& ADM & $6.7371 \times 10^{-8}$ \\
0.0005 & I1P & $2.0798 \times 10^{-11}$ \\
& ADM & $8.3954 \times 10^{-9}$ \\
0.0001 & I1P & $3.3262 \times 10^{-14}$ \\
& ADM & $6.6988 \times 10^{-11}$ \\
\hline
\end{tabular}

Table 4. Numerical Results for Problem 4

\begin{tabular}{ccc}
\hline$h$ & Method & Max \\
\hline 0.05 & I1P & $7.1950 \times 10^{-5}$ \\
& ADM & $8.6247 \times 10^{-4}$ \\
0.01 & I1P & $5.8381 \times 10^{-8}$ \\
& ADM & $5.8075 \times 10^{-6}$ \\
0.005 & I1P & $3.1889 \times 10^{-9}$ \\
& ADM & $6.9849 \times 10^{-7}$ \\
0.001 & I1P & $5.2176 \times 10^{-12}$ \\
& ADM & $5.7040 \times 10^{-9}$ \\
0.0005 & I1P & $3.2163 \times 10^{-13}$ \\
& ADM & $7.1027 \times 10^{-10}$ \\
0.0001 & I1P & $5.1064 \times 10^{-16}$ \\
& ADM & $5.7803 \times 10^{-12}$ \\
\hline
\end{tabular}

Table 5. Numerical Results for Problem 5

\begin{tabular}{lcc}
\hline$h$ & Method & Max \\
\hline 0.05 & I1P & $1.3218 \times 10^{-1}$ \\
& ADM & $1.5547 \times 10^{0}$ \\
0.01 & I1P & $2.2950 \times 10^{-4}$ \\
& ADM & $1.9978 \times 10^{-2}$ \\
0.005 & I1P & $1.4490 \times 10^{-5}$ \\
& ADM & $2.6283 \times 10^{-3}$ \\
0.001 & I1P & $2.3373 \times 10^{-8}$ \\
& ADM & $2.1882 \times 10^{-5}$ \\
0.0005 & I1P & $1.4623 \times 10^{-9}$ \\
& ADM & $2.7487 \times 10^{-6}$ \\
0.0001 & I1P & $2.3415 \times 10^{-12}$ \\
& ADM & $2.2392 \times 10^{-8}$ \\
\hline
\end{tabular}

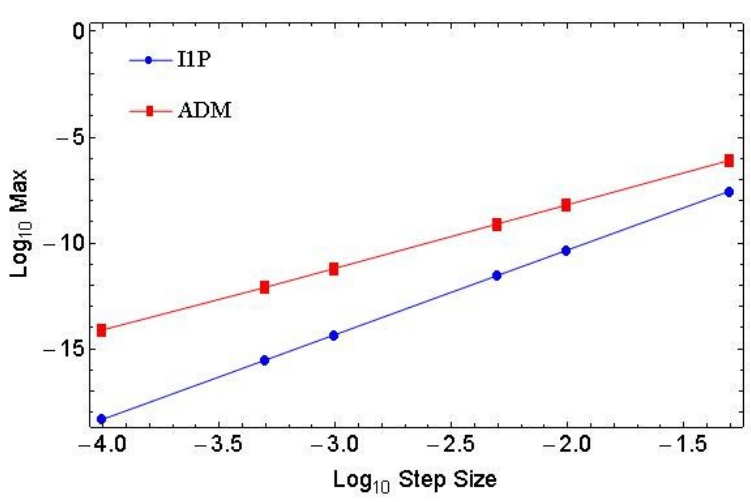

Figure 2. Efficiency curve for Problem 1 


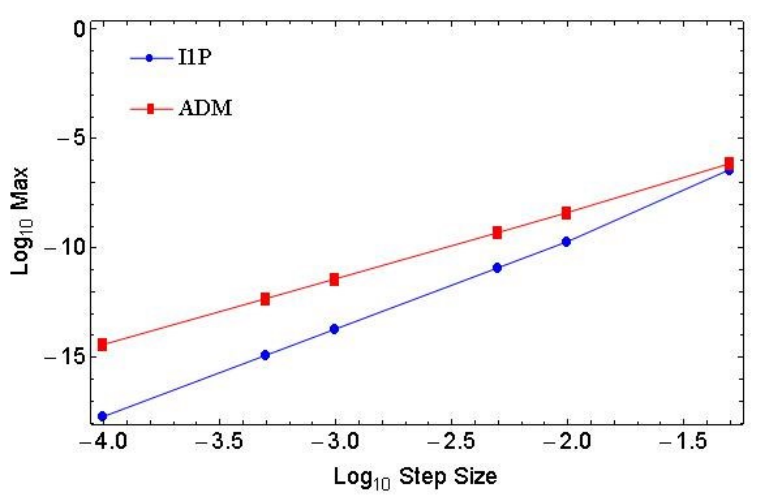

Figure 3. Efficiency curve for Problem 2

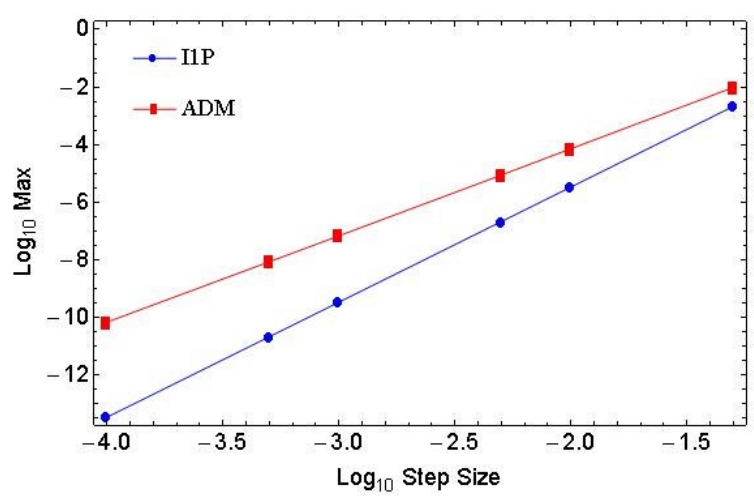

Figure 4. Efficiency curve for Problem 3

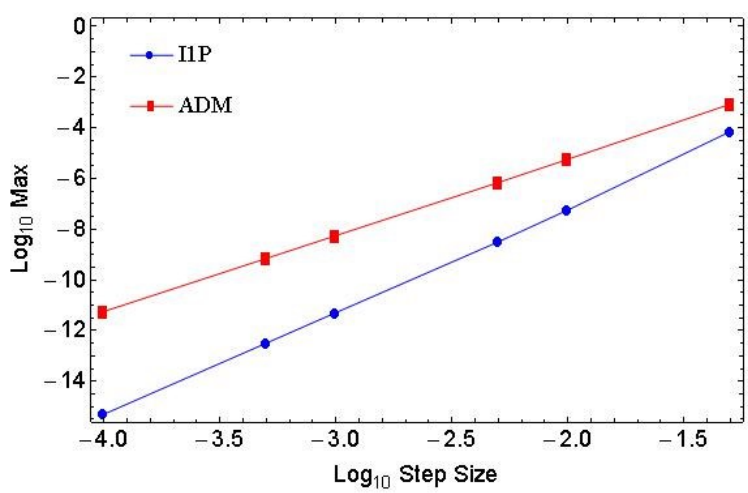

Figure 5. Efficiency curve for Problem 4

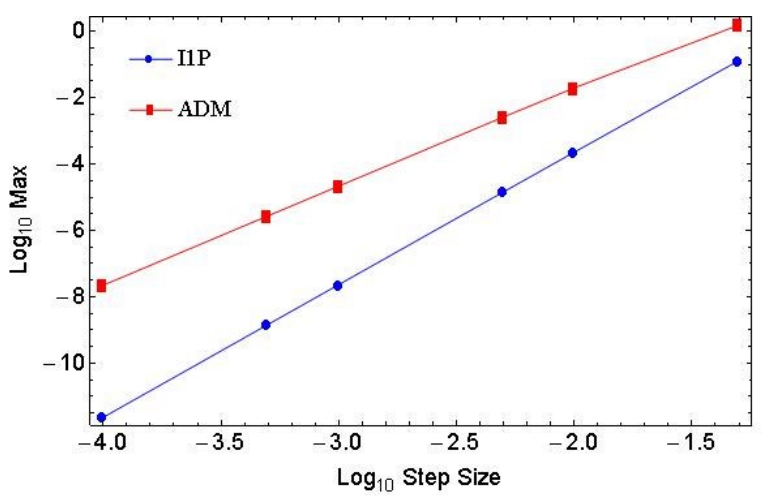

Figure 6. Efficiency curve for Problem 5

\section{DISCUSSION AND CONCLUSION}

The numerical results in Tables 1 to 5 demonstrate that the implicit block hybrid-like method outperforms the existing Adams method of same order when solving systems of first order ordinary differential equations. The efficiency curves in Fig. 2 to 6 also clearly show that the newly proposed method is more efficient compared to existing Adams method. It is apparent that the block hybrid-like method manages to achieve better accuracy as the step size getting smaller. It can also be deduced that the inclusive of off-step point to find the approximation of $y$ at the main point helps to improve the accuracy.

As a whole, we have presented the block hybrid-like method that can be used on wider interval and demonstrates the superiority in providing higher order of accuracy for systems of first order ordinary differential equations.

\section{REFERENCE}

1. Abasi N., Suleiman M., Abbasi N. and Musa H. (2014). 2-Point Block BDF Method with Off-Step Points for Solving Stiff ODEs, Journal of Soft Computing and Applications, Vol. 2014, pp. 1-15.

2. Akinfenwa O. A., Jator S. N. and Yao N. M. (2013). Continuous block backward differentiation formula for solving stiff ordinary differential equations, Computers and Mathematics with Applications, Vol. 65, pp. 996-1005. 
3. Akinfenwa O. A., Yao N. M. and Jator S. N. (2011). Implicit two step continuous hybrid block methods with four off-steps points for solving stiff ordinary differential equation, World Academy of Science, Engineering and Technology, Vol. 75, pp. 425-428.

4. D'Ambrosio R., Esposito E. and Paternoster B. (2012). Exponentially fitted two-step Runge-Kutta methods: construction and parameter selection, Applied Mathematics and Computation, Vol. 218, pp. 7468-7480.

5. D'Ambrosio R. and Paternoster B. (2014). Exponentially fitted singly diagonally implicit Runge-Kutta methods, Journal of Computational and Applied Mathematics, Vol. 263, pp. 277-287.

6. Chapra S. C. and Canale R. P. (2006). Numerical methods for Engineers, 5th ed. Singapore: Mc Graw Hill.

7. Enright W. H. and Higham D. J. (1991). Parallel defect control, BIT, Vol. 31, pp. 647-663.

8. Estep D. (1995). A posteriori error bounds and global error control for approximation of ordinary differential equations, SIAM Journal on Numerical Analysis, Vol. 32, pp. 1-48.

9. Ibrahim Z. B., Othman K. I. and Suleiman M. (2007). Implicit $r$-point block backward differentiation formula for solving first-order stiff ODEs, Applied Mathematics and Computation, Vol. $186(1)$, pp. 558-565.

10. James A. A., Adesanya A. O., Odekunle M. R. and Yakubu D. G. (2013). Constant order predictor corrector method for the solution of modeled problems of first order IVPs of ODEs, World Academy of Science, Engineering and Technology International Journal of Mathematical, Computational, Physical and Quantum Engineering, Vol. 7(11), pp. 1072-1076.

11. Lee L. S. (2000). Two and three-point block methods for solving first order ordinary differential equations in parallel, MSc Thesis, University of Putra Malaysia.

12. Majid Z. A., Mokhtar N. Z. and Suleiman M. (2012). Direct two-point block one-step method for solving general second-order ordinary differential equations, Mathematical Problems in Engineering, Vol. 2012, pp. 1-16.

13. Mehrkanoon S., Majid Z. A. and Suleiman M. (2010). A variable step implicit block multistep method for solving first-order ODEs, Journal of Computational and Applied Mathematics, Vol. 233, pp. 2387-2394.

14. Musa H., Suleiman M. B. and Senu N. (2012). Fully implicit 3-point block extended backward differentiation formula for stiff initial value problems, Applied Mathematical Sciences, Vol. 6(85), pp. 4211-4228.

15. Omar Z. and Suleiman M. (2009). Solving first order systems of ordinary differential equations using parallel $r$-point block method of variable step size and order, Chiang Mai Journal of Science, Vol. 36(1), pp. 9-23. 